\title{
L'analyse de la base du crâne dans les premières années de vie, une approche complémentaire du diagnostic et du traitement des classes II et III
}

\section{RÉSUMÉ}

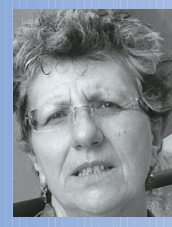

Roselyne LALAUZE-POL*

Ostéopathe DO,

Spécialisé en Ostéopathie Périnatale

et Pédiatrique,

Service de Stomatologie

et de Chirurgie maxillo-faciale

Hôpital Robert Debré,

Paris.

\section{Patrick FELLUS*}

Spécialiste qualifié en ODF,

Attaché consultant des hôpitaux de Paris,

Service de Stomatologie

et de Chirurgie maxillo-faciale

Hôpital R. Debré,

Paris.

\section{Séverine LAMBERT*}

Ostéopathe DO,

Spécialisé en Ostéopathie Périnatale

et Pédiatrique,

Service de Stomatologie

et de Chirurgie maxillo-faciale

Hôpital Robert Debré,

Paris.

Monique ELM ALEH

Radiologiste pédiatrique,

Praticien hospitalier,

Service d'Imagerie Médicale,

Hôpital R. Debré,

Paris.

Selim BENNACEUR*

Chirurgien maxillo-facial,

Plasticien,

Chef de service de Stomatologie et de

Chirurgie maxillo-faciale,

Hôpital R. Debré,

Paris.

* Membre de la SEROPP (Société Européenne de Recherche en Ostéopathie Périnatale et Pédiatrique).
L'analyse basicrânienne de Delaire[1] est une aide au diagnostic d'orthopédie dento-faciale.

L'examen scannographique selon de nouvelles coupes tangentes au plan basicrânien nous a permis de mieux appréhender les conditions biomécaniques de modification de ces angles.

La prise en charge ostéopathique précoce permet une correction durable et efficace de certaines dysmorphoses basicrâniennes et dento-faciales (avant 3 ans). Conjointe au traitement ODF, elle permet d'en réduire la durée. 


\section{Introduction}

$>$

Dès 1965, Delaire[2] a montré que nombre des dysmorphoses faciales squelettiques étaient liées à des dysmorphoses basi-craniennes présentes dès la naissance, ces dernières étant liées aux contraintes subies in utero[2], et/ou per partum[3, 4, 5]. Plus tard, son analyse tridimensionnelle a démontré que les classes II et III étaient corrélées avec la valeur sphénoïdale (N-S-Ba)[1] à l'âge orthodontique.

\section{Développement}

Nous nous intéresserons principalement à l'angle sphénoïdal bien que l'étude du rachis cervical supérieur - RCS - soit complémentaire.

\section{Angle sphénoïdal,}

\section{valeurs au cours \\ de la croissance}

L'angle sphénoïdal est mesuré dans le plan sagittal, il relie 3 points, définissant deux segments, qui, s'ils sont situés dans la même région, ne sont pas strictement localisés aux mêmes repères anatomiques :
Très précocement, il serait donc intéressant de connaître les déterminants de la variation de cet angle, et de pouvoir définir quels sont les facteurs prédictifs conduisant à ces modes pathologiques de croissance.

En s'appuyant sur des analyses cliniques et scannographiques de l'angle sphénoödal dans les premières années de vie, nous présentons I'aspect biomécanique de la base crânienne et ses relations craniofaciales.

- l'angle de Welcker : nasion - tubercule sphénoïdal antérieur/tangente au plan postérieur du basion occipital, est plutôt utilisé par les radiologues ;

- l'angle de Hanzel : nasion - centre de la selle turcique/tangente au plan postérieur du basion occipital[9] ;

- l'angle de Delaire : nasion - sommet de la clinoïde postérieure / tangente au plan postérieur de l'odontoïde; M. Delaire y ajoute les tangentes au plan postérieur du basion sphénoïdal et du basion occipital, ces 2 tangentes doivent normalement être confondues; ces

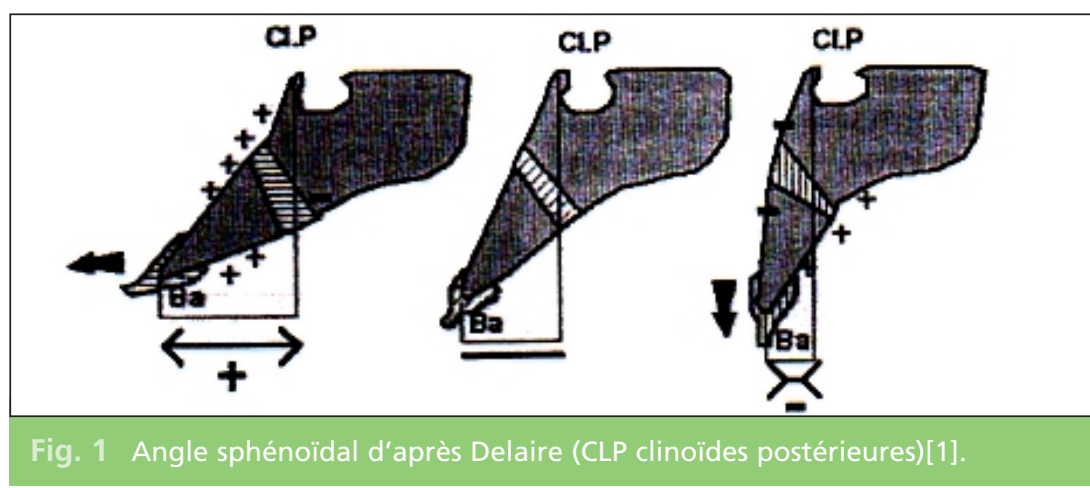


angles sont utilisés par la plupart des orthodontistes (fig. 1) ;

- d'autres angles (Fernex, Downs) existent, avec des points sensiblement différents.

Selon les mesures de Lichtenberg[10], I'angle sphénoïdal, très ouvert en période fœtale $\left(140,7^{\circ}\right.$ à 10 jours) va progressivement se fermer : de $138,26^{\circ}$ à la naissance à $134,55^{\circ}$ vers un an (à la marche). Selon Deshayes[11] sa valeur moyenne est de $116,42^{\circ}$ pour la moyenne populationnelle entre 3 à 6 ans en denture temporaire.

Dans les premiers mois de vie, cet angle peut être influencé par la posture de sommeil. Selon l'action de la gravité, Huang[12] a montré qu'une position de sommeil inchangée (décubitus dorsal - DD - strict ou décubitus latéral - DL - strict) va modifier à vie l'indice crânien. Le DD ferme l'angle basicranien et ouvre l'angle bi-pétreux, le DL ouvre N-S-Ba et ferme l'angle bi-pétreux[1].

Les classes II, très fréquentes en occident (65\%), comme les classes III peuvent être souvent associées à des troubles transversaux couplés à une asymétrie faciale, parfois présente

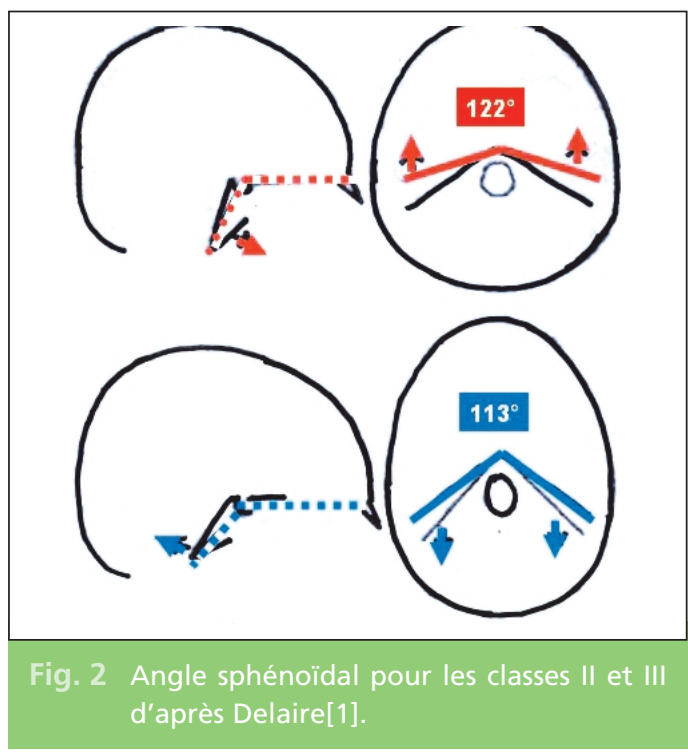

dès la naissance, et/ou à une plagiocéphalie posturale d'origine positionnelle (POPP)[5].

Les dysmorphoses de classe III (prévalence moins de $5 \%$ en occident) se répartissent en rétrognathisme maxillaire, en prognathisme mandibulaire, ou combinent les deux dysmorphoses. Certaines de ces dysmorphoses sont sous dépendance génétique. Dernièrement, Raberin[12] a suggéré que la stabilité du traitement précoce des classes III squelettiques était dépendante de l'angle de la base du crâne, de la morphologie mandibulaire et de la typologie verticale.

La classe III squelettique, comme la classe II, ne se limite pas à la simple dysmorphose des arcades dentaires mais s'intègre dans un cadre plus complexe incluant le développement adaptatif de la base crânienne et de la croissance craniofaciale[1, 11, 14] (fig. 2).

Tollero et al[15] (étude portant sur 60 enfants entre 4 et 6 ans) a montré que dans les classes III, la partie antérieure du crâne est réduite, alors que la partie postérieure est augmentée, à l'inverse de la classe II.

Deshayes[11] (118 enfants), constate que I'angle sphénoïdal est plus fermé pour les enfants dérivant en classe III $\left(115,59^{\circ}+/-4,4\right.$ au test de Student) alors que la moyenne est de $117,41^{\circ}$ (+/-4,3 au test de Student) pour la classe II.

La fermeture de l'angle sphénoïdal amène une ouverture de l'angle des rochers induisant une rotation faciale globale antérieure, caractéristique de l'hominisation. Au cours de la verticalisation entre la naissance et les premiers pas, l'angle sphénoödal va se fermer. L'angle des rochers est de $98^{\circ}$ environ à la naissance, il va progressivement s'ouvrir, entre $115^{\circ}$ à $122^{\circ}$ voire $125^{\circ}[1]$, jusqu'à l'âge de 3 ans, puis rester constant après cet âge.

La classe III squelettique est corrélée avec une fermeture importante de l'angle sphénoïdal 
qui s'accompagne d'une ouverture de l'angle bi-pétreux et d'une frontalisation des pétreux, induisant une antériorisation des ATM, l'ensemble propulsant la mandibule vers l'avant[1, $12,15]$. A l'inverse, la classe II squelettique sera associée à un angle sphénoödal ouvert et à un angle bi-pétreux réduit.

Angle sphénoïdal, approche

du service d'orthopédie

et de chirurgie maxillo-faciale de l'hôpital R. Debré

En périnatalité, la base crânienne présente de larges plages de cartilages hyalins: les synchondroses[16, 17]. Dans le dernier trimestre de la grossesse (dans certaines conditions : oligoamnios, malformation utérine, bassin chi- rurgical, macrosomie, etc.) et en fonction du type d'accouchement (particulièrement dans la voie basse naturelle ou avec extraction instrumentale)[4, 18-21] la sphénobasilaire, mais aussi les autres synchondroses basicrâniennes peuvent subir plusieurs types de contraintes.

A J1 ou J2, I'analyse clinique des crânes[3-4, 2223] a montré des corrélations entre les contraintes périnatales (intra-utérines et/ou per partum) et des glissements de la synchondroses sphénobasilaire (SSB), glissements (strains) des basions (occipital et sphénoïdal) I'un par rapport à l'autre, et/ou des compressions, sur tout ou partie, de la SSB[3-4, 23].

Dans les études d'orthopédie dento-faciale, les mesures de l'angle N-S-Ba ont été faites à partir des téléradiographies réalisées à l'âge orthodontique (ODF précoce 3-4 ans, ODF en période pré-adolescente: 10-12 ans)[1, 14], mais rarement dans les trois premières années
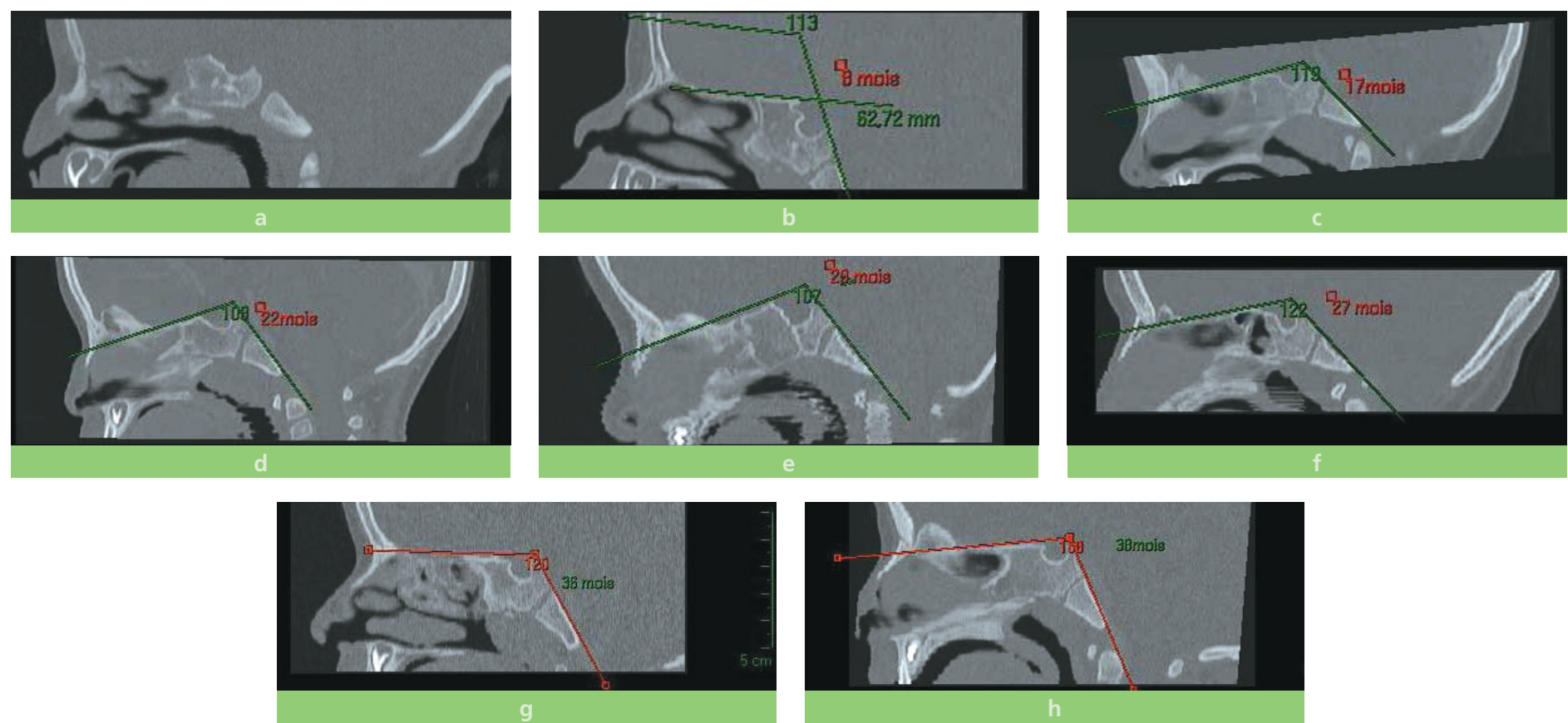

Fig. 3 a à $h$

a : 9 semaines, b : 8 mois, $c: 17$ mois, $d: 22$ mois, e : 27 mois, $f: 29$ mois, $g: 38$ mois, $h: 38$ mois.

Coupes scannographiques sagittales et valeurs de l'angle sphénoïdal en population mixte pendant les premières années de vie. 
de vie. En fonction de la valeur de l'angle, il nous a paru important d'analyser sur le plan biomécanique la SSB, avant l'âge orthodontique. L'analyse scannographique a permis de clarifier notre approche.

Un échantillonnage de coupes sagittales scannographiques réalisées sur des enfants d'une population mixte, ne présentant aucune dysmorphose craniofaciale avérée (mais d'autres pathologies ayant justifiées la prescription d'un scanner) montre que l'angle sphénoïdal (ici de Weckler) peut varier de plus de $10^{\circ}$, à âge égal dans les trois premières années. Une étude plus détaillée, portant sur un grand nombre de sujets, est en cours de réalisation (fig. $3 \mathrm{a}$ à h).

L'analyse scannographique[5] du plan basicrânien nous a permis d'objectiver l'impact biomécanique de ces contraintes périnatales sur la plage cartilagineuse de la SSB.

Les glissements se produisent au sein même du plan sphéno-basilaire dans un sens vertical et/ou latéral (strain vertical ou latéral).

Par convention, le type de glissement (strain) est déterminé par la position du basi-sphénoïde par rapport au basion occipital; par exemple: un latéral strain droit désigne un glissement du basion sphénoïdal vers la droite par rapport au basion occipital[1, 24].

En raison des contraintes inhérentes aux forces de la parturition, les glissements (strains) les plus fréquents sont les suivants :

- glissements latéraux : droit ou gauche ;

- glissement vertical bas : le basion sphénoïdal glisse en bas et en avant par rapport au basion occipital ;

- glissement vertical haut: le basion sphénoïdal glisse en haut et en arrière par rapport au basion occipital.

Le glissement (strain) vertical haut, augmente la fermeture de I'angle N-S-Ba, et semble agrandir la hauteur totale de la lame basilaire (planum), les os pétreux solidaires du basion occipital (par les sutures occipito-pétreuses) se trouvent, de fait, relativement bas, tout comme les ATM (fig. 4).

Le glissement (strain) vertical bas ouvre I'angle N-S-Ba et semble diminuer la hauteur totale du planum, les pyramides pétreuses solidaires du basion occipital et les ATM se trouvant alors sensiblement plus hautes (fig. 5).
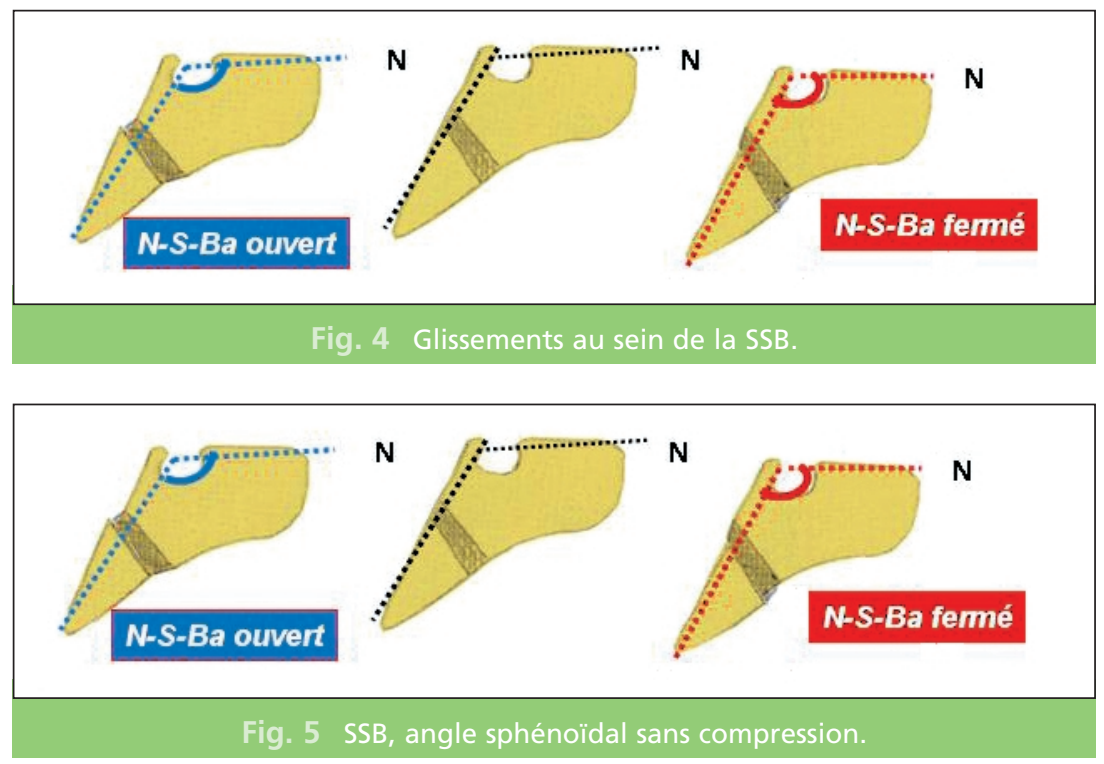

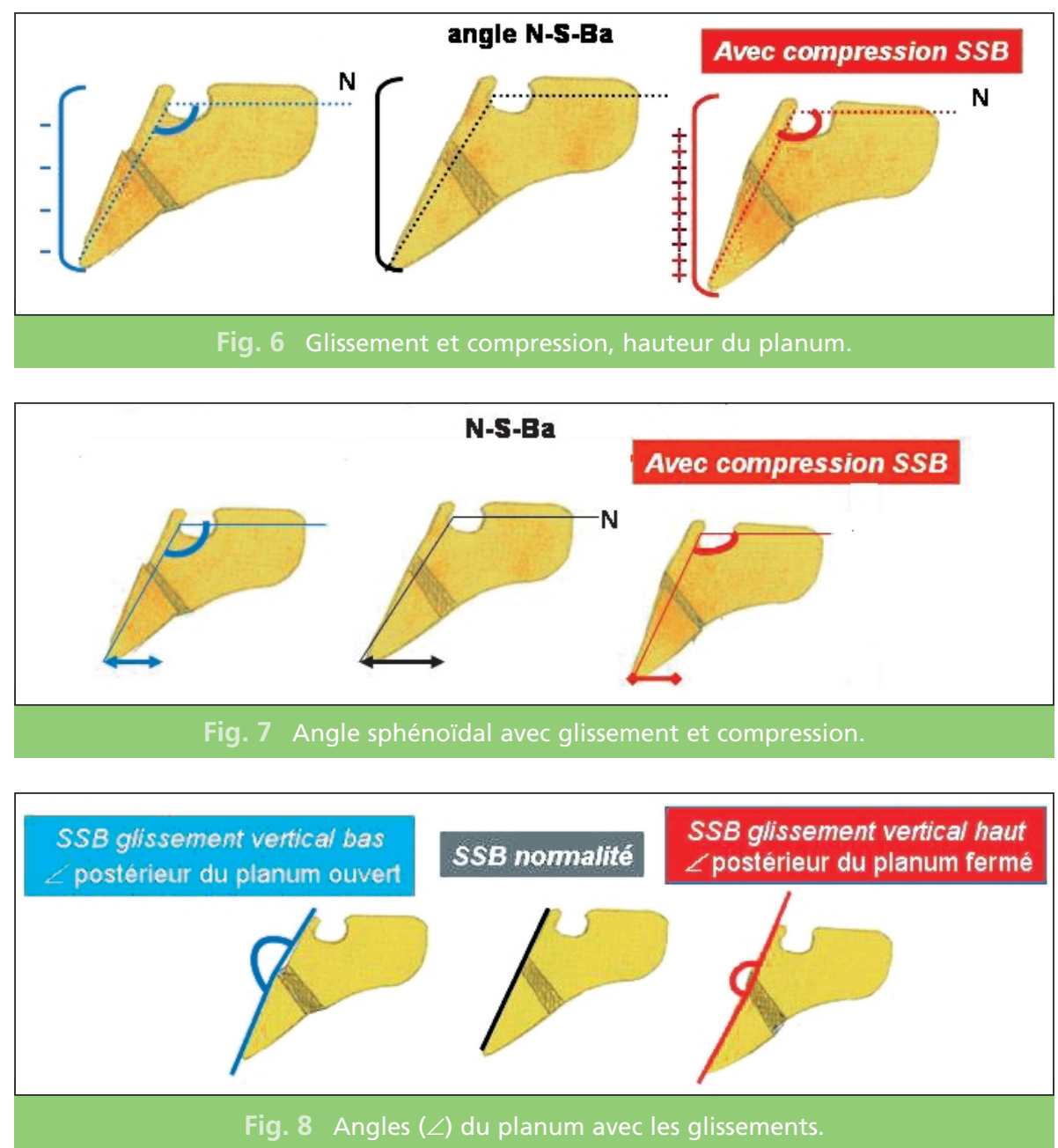

Quand ils sont présents, ces glissements, se combinent très souvent avec une compression (réduction en épaisseur de la plage cartilagineuse) intéressant soit l'ensemble de la SSB, soit unilatéralement, plus fréquemment à droite (en raison de la position fœtale dans l'axe oblique gauche du bassin maternel[3, 4, 25, 26]) (fig. 6) :

- la compression au sein de la SSB, associée à un glissement sphénoïdal vertical haut (vertical up strain), augmente la fermeture de l'angle N-S-Ba (fig. 7) ;

- la compression au sein de la SSB associée à un glissement sphénoïdal vertical (vertical down strain) bas augmente l'ouverture de l'angle N-S-Ba.
Ces glissements vont aussi modifier la ligne postérieure du planum qui va se briser pour former un angle ouvert (glissement bas) et un angle fermé (glissement haut) (fig. 8).

En parallèle, il peut exister des glissements au sein de la selle turcique (synchondroses intrasphénoïdales) et/ou au sein de la sphéno-ethmoïdale, ils sont visibles dans les coupes scannographiques avant leur fermeture dans la première année. De même les sutures occipitopétreuses peuvent subir des contraintes et les cervicales hautes peuvent présenter des dysfonctions dans le plan antéro-postérieur, ou des troubles rotatoires du RCS[4] (fig. 9 et fig. 10). 


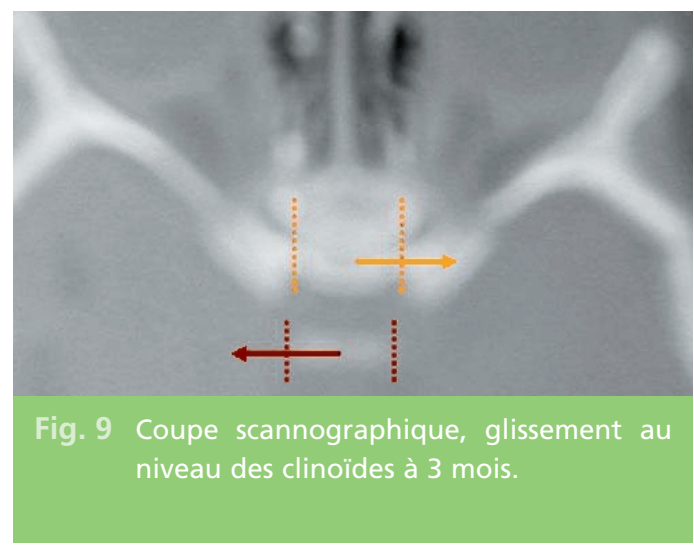

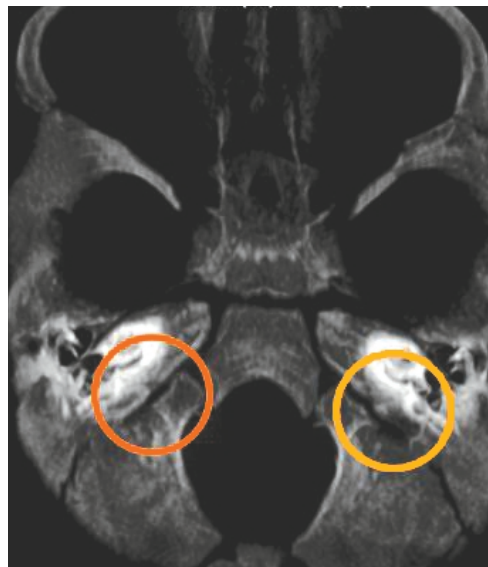

Fig. 10 Coupe scannographique, compression unilatérale occipito-pétreuse.

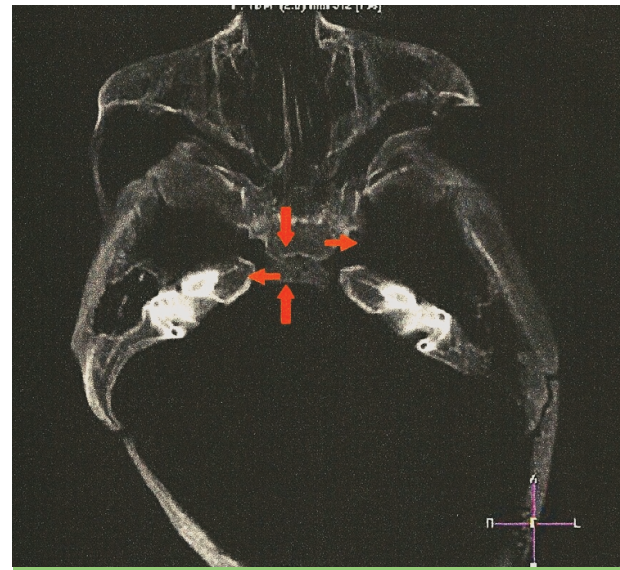

Fig. 11 Coupe scannographique, glissement latéral au sein de la SSB.
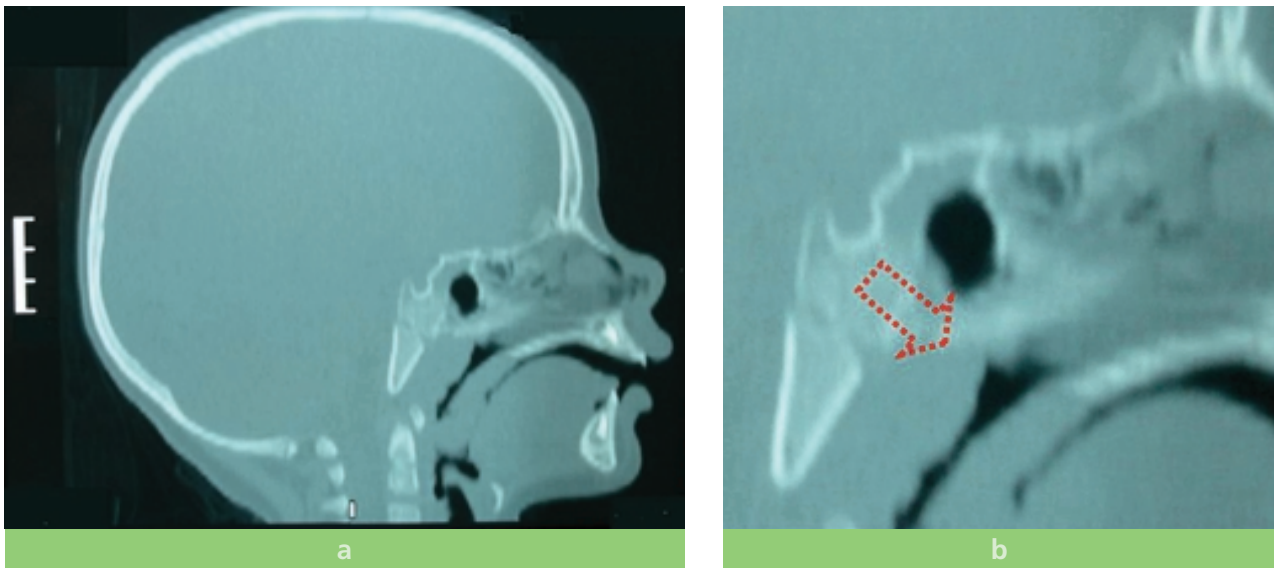

Fig. 12 a et b Coupes scannographiques, glissement vertical bas.

Les glissements au sein de la SSB pourraient être à l'origine de certaines classes squelettiques III (non génétiques) et de classes II. Les troubles de la fonction linguale, de la déglutition et de la mastication seraient alors suscep- tibles, selon les modalités de la mécano-transduction*[27, 28], d'interférer sur la croissance future, en pérennisant, voire en augmentant les dysfonctions basicrâniennes préexistantes[4] (fig. 11 et fig. 12 a et b).

\footnotetext{
* Définition de la mécano-transduction :

Capacité d'adaptation et d'optimisation des cellules aux contraintes subies (compression, torsion, traction...) influençant directement leur fonctionnement (génétique, histologie, biosynthétique, ect.) et entraînant une modification tissulaire.
} 


\section{Conclusion}

Au sein du service, par une approche pluridisciplinaire "ODF et ostéopathie» nous prenons en charge ces différents dysfonctionnements, la prise en charge ostéopathique peut intervenir plus ou moins précocement (fig. 13). Au regard des récentes connaissances scientifiques, il semble que les forces appliquées tant par l'orthodontiste (forces permanentes) que par l'ostéopathe (forces séquentielles et ponctuelles) sur des plages osseuses et/ou cartilagineuses, ont une action mécano-transductrice[27-29] à visée correctrice.
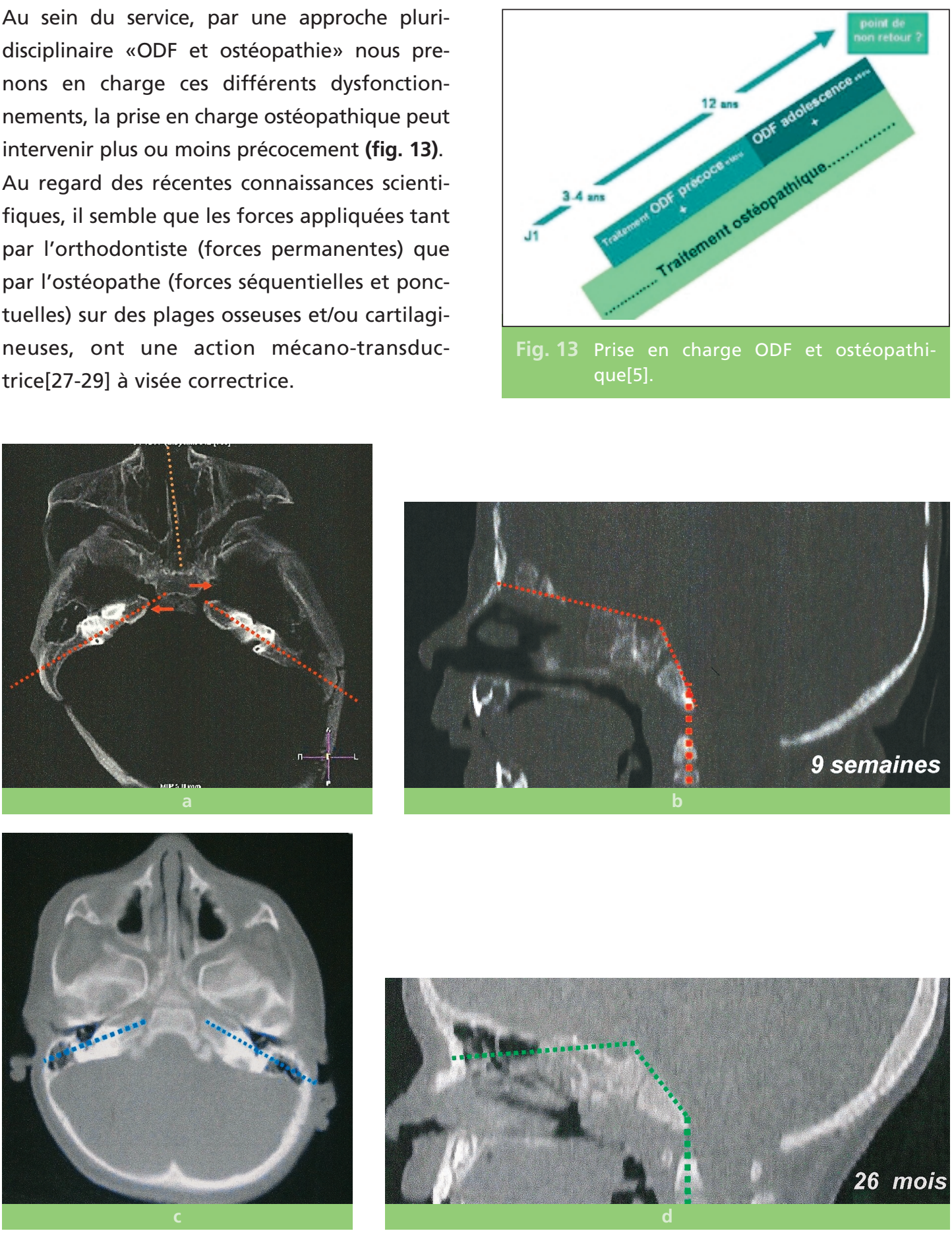

Fig. 14 a à d Coupes scannographiques, changement de l'angle sphénoïdal, entre 9 semaines lors de la prise en charge ostéopathique (compression, glissement latéral, luxation rotatoire du RCS (14 a et 14 b) et 26 mois ( 7 consultations en 19 mois) (14 c et 14 d)[5]. 
Suite à un accouchement dystocique, la prise en charge ostéopathique se fera[3-5, 13, 23, 30] dès les premiers jours de vie. Cependant cette pratique ne peut être suffisante, dans les six premiers mois de vie (angle sphénoïdal est dépendant de la position de couchage)[12], la coopération parentale est aussi détermi- nante. Il faudra aménager le couchage (notion de sécurité) en modifiant les habitudes, avec comme support une fiche "conseils au parents» adaptée au nourrisson (réalisée dès 2003, en collaboration avec le service d'orthopédie pédiatrique de $\mathrm{R}$. Debré) est remise aux parents[31] (fig. 14 a à d).

\section{Abréviations}

ATM : articulation temporo-mandibulaire

CLP : clinoïdes postérieures

DD : décubitus dorsal

$\mathrm{DL}$ : décubitus latéral

J1 : premier jour de vie

$\mathrm{J} 2$ : deuxième jour de vie
$\mathrm{N}-\mathrm{S}-\mathrm{Ba}$ : angle nasion-sphénoïde-basiocciput ODF : orthopédie dento-faciale

RSC : rachis cervical supérieur

SSB : synchondrose sphénobasilaire

$\angle$ : angle

\section{Bibliographie}

1. Delaire J.

Le développement

adaptatif

de la base du crâne.

Justification

du traitement précoce

des dysmorphoses

de classes III.

Rev Orthop Dento-Faciale

2003;37:243-265.

2. Delaire J, Billet J, Ferré JC, Julia P.

Malformation faciale

et asymétrie de la base

du crâne

(un nouveau syndrome

malformatif intéressant

l'orthodondiste).

Revue de Stomatologie

1965;66:379-396.
3. Lalauze-Pol $\mathrm{R}$,

Mendizabal $\mathrm{H}$,

Leclaire $M$, Unal D.

Prévalence des impactions

des synchondroses

et des chevauchements

des sutures crâniennes

du nouveau-né

à J1 ou J2 (312 cas).

Marseille ; CHU-Conception, 1998 (non publié).

4. Lalauze-Pol R.

Le crâne du nouveau-né.

Monpellier :

Sauramps-médical

2003:195-197,206-207, 934-936.

5. Lalauze-Pol $R$, Lambert $S$, Fellus $\mathrm{P}$, Bennaceur $\mathrm{S}$,
Elmaleh M.

La prise en charge précoce

de la plagiocéphalie,

une prévention

des dysmorphoses

maxillofaciales.

Rev Orthop Dento Faciale

2007;41(2):125-139.

6. Klocke L, Nanda RS,

Kalh-Nieke B.

Role of cranial base

flexure

in developing jaw

discrepansies.

Am J Orthod Dentofacial

Orthop 2002;(122):386-391.

7. Dambricourt Malassé A.

Un nouvel angle

de la base 
pour une meilleure

compréhension

de la morphogénése

basicrânienne

des hominidés

et des modalité évolutive.

Biom Hum Et Antropol

2005;23, 1-2:53-62.

8. Deshayes MJ.

Mécanique crânienne

et morphogenèse

mandibulaire.

Étude des effets

d'un "forçage"

par déformation

intentionnelle

(1re partie).

Biom Hum Et Antropol

2005;23,1-2:83-93.

9. Hanzel B.

L'incidence

des déformations

crâniennes

sur le massif facial.

Analyse de 48 crânes

paléo-péruviens déformés.

Thèse de doctorat d'état

de sciences naturelles.

Paris V 1979:294-1;

10. Lichtenberg $\mathrm{R}$.

Radiographie du crâne de 228 enfants normaux de la naissance à 8 ans.

Thèse pour le Doctorat en médecine.

Paris 1960:86.

11. Deshayes MJ, Desvignes $M$, Romaniuk J, Revenu M,

Deshayes B.

Une analyse crânienne

au service

des traitements précoces

et du concept

biodynamique

de la morphogenèse

cranio-faciale.

Orthod Fr 2002;Dec;73(4):

395-406.

12. Huang $\mathrm{CS}$, Cheng $\mathrm{HC}$, Lin Wy, Liou JW,
Chen YR.

Skull morphology

affected by different

sleep position

in Infancy.

Cleft Palate-craniofacial J

1995;32(5):413-419.

13. Raberin $M$, Morgon $L$,

Gay-Brevet K.

Facteurs décisionnels

céphalométriques

dans les traitements

précoces des classes III

squelettiques.

Orthod Fr 2007;78:101-112.

14. Deshayes MJ.

L'art de traiter

avant 6 ans.

Ed. Cranexplo 2006:157-210.

15. Tollaro I, Baccetti V,

Francheschi L.

Class III malocclusion

in the deciduous dentition:

morphological

and correlation study.

Eur J orthod 1994;16:401-08.

16. Belden CJ.

Skull base and calvaria, adult and pediatric.

Neuroimaging clin $\mathrm{N} \mathrm{Am}$

1998 Feb;8(1):1-20.

Review.

17. Elmaleh M, Sekkal A,

Hassan M.

Aspect en imagerie

du sphénoïde

au cours de la croissance.

J Neuroradiol

2003;30:249-257.

18. Barbarino-Monnier $P$,

Boutroy JL.

Présentations du siège.

In Schaal JP.

Riethmuller D

Maillet R.

Mécanique

et techniques

obstétricales.

Sauramps 2e éd. 1998

nov:233-8.
19. De Tourris $H$, Henrion $R$,

Delecour $\mathrm{M}$.

Abrégé illustré

de gynécologie

et obstétrique.

Masson $5^{\mathrm{e}}$ ed 1984:100-4.

20. Hannah ME, Hannah WJ, Hewson SA, Hodnett ED, Saigal S, Willan AR.

Planned caesarean

section versus

planned vaginal birth

for breech presentation

at term: a randomised

multicentre trial.

Term Breech

Trial Collaborative Group.

Lancet 2000; Oct 21;

356(9239):1375-83.

21. Rothenberg SJ, Schaas $L$, Perroni E, Herandez RM, Martinez S, Hernandez C.

Pre-and postnatal

lead effect

on head circumference:

a case for critical period.

Neurooxicol Teratol

1999:21(1):1-11.

22. Lalauze-Pol $\mathrm{R}$,

Mendizabal $\mathrm{H}$,

Delarozière $\mathrm{JC}$.

Is there any relationship

between impactions,

overlapping,

dysmorphisms

of skull seams

and some neurovegetative

troubles or anomalies

locomotion system.

Unconventional medecine

at beginning

of third millennium.

Pavia: European Commission

COST B4 1998:44.

23. Lalauze-Pol R.

L'ostéopathie

en maternité

et en néonatologie:

diminuer

les contraintes subies

par le fœtus. 
Dossiers d'obstétrique 2005;(342):19-22.

24. Magoun HI. Ostéopathie dans le champs crânien. Edition originale 1951 ; vanes; Edition Sully 2000.

25. Mac Grahams. Smith's recognizable Patterns of Human Deformation. Philadelphia : WB. Saunders Company $2^{\text {nd }}$ ed, 1988:162.

26. Mac Grahams. Le point de vue du dysmorphologiste.
In: Amiel-Tison C,

Stewart A.

L'enfant nouveau-né, un cerveau pour la vie. Paris : INSERM 1995:27-43.

27. Portet $\mathrm{S}$.

Mécanotransduction.

CNAM -

Probatoire

de Calcul Scientifique www.cnam.fr/math/IMG/pdf/

Probatoire

CelluleEtModelisation.pdf.

28. Mao JJ.

Mechanobiology

of craniofacial sutures.

J. Dent. Res. 2002;81(12):

810-16.
29. He T.

Craniofacial

morphology

and growth

in the ferret:

effects from alteration

of masticatory function.

Swed Dent J Suppl

2004;165:1-72.

30. Amigues JP.

Le système

stomatognatique,

concept orthodontique, concept ostéopathique.

Montpellier :

Sauramps-médical 2004:234.

31. http://www.osteopathieperinatale-pediatrique.org/

\section{SUMMARY}

\section{Analysis of the cranial base in early years of life, a complementary approach of the diagnostic and treatment of class II and III}

Roselyne LALAUZE-POL, Patrick FELLUS,

Séverine LAMBERT,

Monique ELMALEH,

Selim BENNACEUR

\footnotetext{
Keywords

- basilar dysmorphosis

- basilar synchondrosis

- early orthopedic

treament

- maxillo-facial

dysmorphosis

- osteopathy

- petrus bone
}

The basilar analysis of Delaire[1] is an aid for the diagnosis of dentofacial orthopedics. Examination by CTscan with new transverse section of the basilar synchondrosis has enabled us to better understand the modifications of biomechanical conditions of the basi-cranial angles. Early osteopathic care allows durable and effective corrections of some basilar and maxillo-facial dysmorphosis (before the age of 3). In association with an dento-facial orthopedics treatment, it also reduces its duration. 


\section{Association d'Enseignement d'Odontologie et de Stomatologie}

\section{LA VIE DE L'ASSOCIATION}

PRÉSIDENTS D'HONNEUR :

Pierre CERNÉA', Jean OUVRARD ${ }^{\dagger}$, Michel BENOIST ${ }^{\dagger}$

\section{MEMBRES D'HONNEUR :}

A. RICHARD ${ }^{\dagger}$, H. LENTULO',

A. LAMBERT, P. GONON ${ }^{\dagger}$,

J.-P. RAGOT'

Ch. DESCROZAILLES,

G. FICHELLE

\section{CONSEIL D'ADMINISTRATION}

PRÉSIDENT : Hubert OUVRARD

VICE-PRÉSIDENTS :

LUC CHIKHANI,

Jean-François LEGRAND

SECRÉTAIRE GÉNÉRAL :

Frédéric LARCHÉ

TRÉSORIER :

Gérard PASQUET

MEMBRES :

Jacques-Charles BERTRAND, Danielle BUCH, Jean BUQUET, Estelle FAVRE, Jean MEYER,

Guy PRINC, Valérie TRAVERT

\begin{abstract}
COMITÉ SCIENTIFIQUE
PRÉSIDENT : LUC CHIKHANI

VICE-PRÉSIDENT : Hubert OUVRARD

SECRÉTAIRE : Frédéric LARCHÉ

MEMBRES : Christophe BONNEFOY, Estelle FAVRE, Gilles FLEURIDAS, Thierry GUÉRIN, Jean-François LEGRAND, Frank LEVAVASSEUR, Hervé MISSISTRANO, Gérard PASQUET, Thierry PIRAL, Valérie TRAVERT
\end{abstract}

ANCIENS PRÉSIDENTS : P. CERNÉA ${ }^{\dagger}$, P. HENNION, H. LENTULO ${ }^{\dagger}$, M. LEPOIVRE ${ }^{\dagger}$ A. MARMASSE ${ }^{\dagger}$, A. LAMBERT, R.-R. RIGOLET ${ }^{\dagger}$, R. BATAILLE', L.-A. STIEGLER ${ }^{\dagger}$, F. BOUCHON ${ }^{\dagger}$, Ch. DESCROZAILLES, P. FRIEZ ${ }^{\dagger}$, F. BROCHERE ${ }^{\dagger}$, A. RICHARD ${ }^{\dagger}$, R.-L. NINET ${ }^{\dagger}$, M. CHATEAU, P. COUSTAING, A. MUGNIER, R. WEILL ${ }^{\dagger}$,

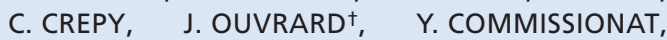
B. DANGY ${ }^{\dagger}$, F. GARLOPEAU ${ }^{\dagger}$, J.-L. DEPHILIPPE, J. VIGNEUL, H. PETIT, M. BENOIST ${ }^{\dagger}$, G. FICHELLE, J. LAUFER, J.-P. SANTORO, P. LAUDENBACH ${ }^{+}$, P. DARGENT ${ }^{\dagger}$, CI. SCHUHMANN, F. MAESTRONI ${ }^{\dagger}$, J.-P. DEFFEZ, H. OUVRARD, D. RIGOLET, J. BUQUET, J.$\begin{array}{lll}\text { P. RAGOT } & \text { R. BUGUGNANI, P. BORDAIS, }\end{array}$ J.-M. LAURICHESSE', J.-Ch. BERTRAND, J.-F. LEGRAND, G. PASQUET, J. DICHAMP, D. BUCH, A. DEBOISE ${ }^{\dagger}$, C. BOZON, F. LARCHÉ, M. AMORIC, G. PRINC, V. TRAVERT, J.-P. LÉZY, F. LEVAVASSEUR, E. FAVRE, H. MISSISTRANO, T. PIRAL, C. BONNEFOY

\section{BULLETIN D'ADHÉSION À L'A.E.O.S.}

$\square$ Oui, je souhaite devenir membre de l'A.E.O.S. Ci-joint le règlement de ma cotisation annuelle $2009: 20 €$ au lieu de $60 €$ (offre réservée aux nouveaux abonnés des A.O.S.).

Je règle par chèque à l'ordre de l'A.E.O.S.

Dr:

Prénom :

Adresse

Code postal :

Ville :

Téléphone :

Fax :

Bulletin à retourner à : I'Association d'Enseignement d'Odontologie et de Stomatologie (A.E.O.S.) 179, rue Saint-Honoré - 75001 Paris - Tél. 0142605032 - Fax 0147034643 